\title{
Cross-chirality transfer of kinetic energy and helicity in compressible helical turbulence
}

\author{
Zheng Yan $\odot$, Xinliang Li $\odot{ }^{*}$ and Changping $\mathrm{Yu}^{\dagger}$ \\ LHD, Institute of Mechanics, Chinese Academy of Sciences, Beijing 100190, China \\ and School of Engineering Science, University of Chinese Academy of Sciences, Beijing 100049, China \\ Jianchun Wang $(1)$ \\ Shenzhen Key Laboratory of Complex Aerospace Flows, Center for Complex Flows and Soft Matter Research, \\ Department of Mechanics and Aerospace Engineering, Southern University of Science and Technology, \\ Shenzhen, Guangdong 518055, China
}

(Received 9 May 2020; accepted 22 July 2020; published 10 August 2020)

\begin{abstract}
As an underlying mechanism, cross-chirality transfer of kinetic energy and helicity plays an essential role in the turbulent dynamics, which is as important as cross-scale transfer especially in broken mirror-symmetry turbulence. The effects of helicity on the properties of turbulent flows in previous studies highlight the role of cross chirality, which may be developed into an efficient method of turbulence control. We numerically study the cross-chirality transfer of kinetic energy and helicity in this paper, particularly under the influence of compressibility in stationary helical homogeneous and isotropic turbulence. Through combining the Helmholtz decomposition and helical wave decomposition, a general helical wave decomposition is proposed to provide three orthogonal bases for velocity. Within the scope of chiral helicity, there also exists chiral kinetic energy based on chiral velocity. They are defined as the left- and right-chirality kinetic energy, and the remaining compressible component of velocity corresponds to free-chirality kinetic energy. Although there exists no difference in the definition of helicity in incompressible and compressible turbulence, its space-time evolution equation in compressible turbulence involves the compressible component of velocity. The compressibility has a great influence on the homochiral kinetic energy and helicity cascade, and it also plays an essential role in the chirality transfer process like cross-chirality kinetic energy and helicity transfer. The amplitude of cross-chirality kinetic energy transfer is comparable with cross-scale kinetic energy transfer at relatively large scales, and also with viscous dissipation at relatively small scales. The triple nonlinear interactions dominate the cross-chirality transfer relative to pairwise interaction of chiral modes, and it is less sensitive to compressibility. Relative to the compression, the expansion of fluid elements can lead to inverse chirality transfer and strengthen the lack of mirror symmetry. The only discrepancy of cross-chirality helicity transfer between incompressible and compressible turbulence lies in the medium role of the compressible component of velocity. The helicity transfer via the compressible component is weak, and even the kinetic energy of the compressible component relative to that of the other two chiral modes is highest. In addition, the inverse helicity transfer is always statistically associated with the compressible component of velocity via triple interaction extracted from nonlinear interactions.
\end{abstract}

DOI: 10.1103/PhysRevFluids.5.084604

\footnotetext{
*1ixl@imech.ac.cn

†cpyu@imech.ac.cn
} 


\section{INTRODUCTION}

Compressible helical turbulence plays an important role in the areas of high-performance aircraft engines, high-speed vehicles, and inertial confinement fusion. Considering the theoretical demands of some extreme phenomena such as supernova explosions, the warm interstellar medium, and the intracluster medium between galaxies [1,2], it is essential to explore the complex characters of compressible turbulent dynamics under the influence of apparently broken mirror symmetry. The broken mirror symmetry exists approximately in almost all of the turbulent flows. The local density of helicity may be large enough to simultaneously affect the properties of compressible flows, with production, diffusion, and dissipation $[3,4]$.

The role of helicity in turbulent flows has been a controversial issue in the past few decades, and the properties and behaviours of helicity itself are prerequisites for further exploring the effect of helicity on turbulent flows. Helicity reflects the degree of broken mirror symmetry [5], and it is defined as

$$
H(t)=\int \mathbf{u}(\mathbf{x}, t) \cdot \omega(\mathbf{x}, t) d \mathbf{x}
$$

where $\mathbf{u}(\mathbf{x}, t)$ is the velocity and $\boldsymbol{\omega}(\mathbf{x}, t)$ is the vorticity. The integrand $h(t)=\mathbf{u}(\mathbf{x}, t) \cdot \boldsymbol{\omega}(\mathbf{x}, t)$ is called the density of helicity [6]. Relative to kinetic energy, helicity measures the degree of knottedness or linkage of vortex lines $[7,8]$. The conservation law of helicity was found by Moreau [9] in 1961, and the hypotheses were the same as those of Helmholtz theorem. To date, the discovery is regarded as the latest milestone of ideal fluid flows governed by Euler's equations [10]. According to the Noether theorem, the conservation law of physical variables determines the evolution of the system [11]. Hence, it is essential to explore the properties of helicity, as we may be able to theoretically prove that there do not exist any other inviscid invariants in three-dimensional flows besides kinetic energy and helicity $[12,13]$. The properties of Euler equations reveal a joint cascade of energy and helicity in three-dimensional turbulent flows [14,15]. Incompressible helical turbulence has always been an important point when exploring the physical mechanism of a joint turbulent cascade in homogeneous and isotropic turbulence (HIT), rotating turbulence, and the boundary layer of atmospheric turbulence in the past few years $[6,11,16,17]$. There have been a few studies to determine the spatial and time scales of turbulence based on helicity $[18,19]$. Some turbulence modelings are developed based on the evolution equation of helicity for large eddy simulations (LESs). Li et al. [20] added several two-term helical modes based on rates of energy and helicity cascade to the subgrid-scale (SGS) modeling in LESs, and coefficients were determined dynamically. Yu et al. [21], Zhou et al. [22] proposed a novel SGS model based on helicity dissipation balance theory for transition and turbulent flows. Recently, Yokoi and Yoshizawa [23] put forward an SGS model with a structural effect incorporated through helicity analogous to the Reynolds-averaged turbulence model. The role of helicity of this model is to suppress "too dissipative" properties of Smagorinsky-type model.

In a compressible case, whether the fluid is barotropic determines the constancy of helicity $[13,24,25]$. The barotropy means that the pressure is the only function of the density [24]. As for compressible Euler equations, we can obtain the helicity governing equation

$$
\partial_{t}(\mathbf{u} \cdot \boldsymbol{\omega})+\operatorname{div}\left\{(\mathbf{u} \cdot \boldsymbol{\omega}) \mathbf{u}+\left[p(\rho)-\frac{1}{2}|\mathbf{u}|^{2}\right] \boldsymbol{\omega}\right\}=0
$$

where $p(\rho)$ is the pressure in the case of barotropic fluid. However, when the shock wave is present, the helicity is not conserved anymore. One paper insisted that the constancy of helicity even facing the presence of shock wave also makes sense [13]. While the conservation characteristics of kinetic energy and helicity in compressible baroclinic turbulence would be no longer satisfied, the presences of nonzero ensemble averages of pressure terms are highlighted as the main reasons in contrast to incompressible turbulence. In the past few years, many efforts have been devoted to generalizing the kinetic energy cascade theory in incompressible turbulence to compressible turbulence [26-28], 
and the existence of conservative energy cascade was clarified in compressible flows. Pressure-work defect and local cascade were regarded as the main reasons for anomalous dissipation of kinetic energy in compressible turbulence [29]. Recently, we also established the helicity cascade theory in compressible turbulence [30]. The main obstacle, the pressure term present in helicity governing equation, was eliminated statistically out of conventional large scales. Hence, the conservative helicity cascade exists statistically in the inertial subrange in compressible turbulence.

The characteristics of homochiral evolutions and chirality transfer were widely explored in the area of magnetohydrodynamic turbulence [6], the production of chiral medicine and pesticides, a single mirror isomer at supramolecular and material levels, the developments of chiral materials, etc. Homochiral and cross-chirality evolutions of kinetic energy and helicity provide many new perspectives to further explore the physical mechanism of turbulent cascade [16,31-33], and the helical wave decomposition (HWD) is a common method to decompose velocity or a vorticity field into different chiralities [31]. Alexakis and Biferale [17] made a comprehensive review, which highlights the importance of chirality in turbulence. Waleffe [31] revealed that the backward energy cascade occurs locally between the same helical signs of velocity and vorticity, and nonlocally integrated with all length scales. Chen et al. [16] argued that the inertial subrange of the helicity cascade is not shorter than a energy cascade through the analysis of net helicity flux. The transfer of energy and helicity between two helical modes corresponds to advection and vortex stretching, respectively. Biferale et al. [33] replaced nonlinear terms in Navier-Stokes equations (NSEs) with the specified helical Fourier basis to carry out well-defined helical direct numerical simulations (DNSs), and demonstrated that the inverse energy cascade is not necessarily related to two-dimensionalization flows. The triadic interactions of the velocity of the same chirality in three-dimensional flows are acceptable widely about the backward energy cascade [32-34]. In addition, the direction of the energy cascade is very sensitive to the presence of different chiralities and could, therefore, change to an infinitesimal amplitude of opposite chirality [35]. The right-chirality helicity cascade in the logarithmic range of boundary layer has been verified by theoretical and numerical investigations in the atmospheric Ekman layer [36,37]. In our previous studies about compressible helical turbulence, we found that helicity can hinder the energy cascade process [38], and it also affects the interchange procedure of kinetic energy and internal energy via a pressure-dilatation term [30].

The effects of helicity on the energy cascade process discussed above highlight the role of helicity transfer, and helicity transfer may be regarded as a controlling method to affect the properties of energy transfer. Until now, few efforts have been devoted to studying the cross-chirality kinetic energy and helicity transfer under the influence of compressibility. In this study, we numerically analyze the influence of complex factors on the joint cross-chirality transfer of kinetic energy and helicity in compressible helical turbulence. We attached emphasis to the local compressibility and straining or rotating regions, and their influences on compressible turbulent dynamics are numerically investigated by conditional averages $[39,40]$. The rest of paper is organized as follows. First, we describe governing equations, our numerical method, and some characteristic parameters of the steady flows. Next, we deduce the governing equations for the filtered kinetic energy and helicity projected on different chiralities. Finally, we discuss the statistical analysis of the cross-chirality kinetic energy and helicity transfer in detail.

\section{GOVERNING EQUATIONS, NUMERICAL METHOD, AND SOME PARAMETERS}

We selected a few reference variables to normalize the variables in the governing equations. These variables included the reference density $\rho_{f}$, velocity $U_{f}$, pressure $p_{f}=\rho_{f} U_{f}^{2}$, temperature $T_{f}$, length $L_{f}$, viscosity $\mu_{f}$, thermal conductivity $\kappa_{f}$, and energy per unit volume $\rho_{f} U_{f}^{2}$. In addition, the three dimensionless parameters chosen are Reynolds number $\operatorname{Re} \equiv \rho_{f} U_{f} L_{f} / \mu_{f}$, Mach number $M \equiv U_{f} / c_{f}$, and Prandtl number $\operatorname{Pr} \equiv \mu_{f} C_{p} / \kappa_{f}$, which is set to 0.7. $c_{f} \equiv \sqrt{\gamma R T_{f}}$ is the speed of sound, where $\gamma \equiv C_{p} / C_{v}$ is the heat capacity ratio, which is set to 1.4 in our numerical simulation, 
$C_{p}$ is the specific heat at constant pressure, $C_{v}$ is the specific heat at constant volume, and another parameter $\alpha \equiv \operatorname{Pr} \operatorname{Re}(\gamma-1) M^{2}$.

The dimensionless NSEs for three-dimensional compressible helical turbulence of ideal gas are

$$
\begin{aligned}
\frac{\partial \rho}{\partial t}+\frac{\partial\left(\rho u_{j}\right)}{\partial x_{j}} & =0 \\
\frac{\partial\left(\rho u_{i}\right)}{\partial t}+\frac{\partial\left(\rho u_{i} u_{j}\right)}{\partial x_{j}} & =-\frac{\partial p}{\partial x_{i}}+\frac{1}{\operatorname{Re}} \frac{\partial \sigma_{i j}}{\partial x_{j}}+F_{i} \\
\frac{\partial \mathscr{E}}{\partial t}+\frac{\partial\left[(\mathscr{E}+p) u_{j}\right]}{\partial x_{j}} & =\frac{1}{\alpha} \frac{\partial}{\partial x_{j}}\left(\kappa \frac{\partial T}{\partial x_{j}}\right)+\frac{1}{\operatorname{Re}} \frac{\partial\left(\sigma_{i j} u_{i}\right)}{\partial x_{j}}-\Lambda+F_{j} u_{j}, \\
p & =\frac{\rho T}{\gamma M^{2}},
\end{aligned}
$$

where $\rho$ is the density, $\mathbf{u}$ is the velocity, $p$ is the pressure, $T$ is the temperature, $\Lambda$ is a coolingfunction for sustaining a steady state statistically, and $\mathbf{F}$ is the large-scale force composed of multiple parameters controlling kinetic energy and helicity. The multiple parameters include the energy inputting rates of compressive and solenoidal components and the helicity inputting rate. Hence, the specific external forcing can be constructed as

$$
F_{i}=\sqrt{\rho} \times\left(\pi_{1} u_{i}^{C}+\pi_{2} u_{i}^{S}+\pi_{3} \omega_{i}\right)
$$

where the superscripts $C$ and $S$ denote the compressible and solenoidal component of velocity, respectively, and $\pi_{1}, \pi_{2}$, and $\pi_{3}$ are three indeterminate dimensional parameters. In order to prevent the flow field from the pollution of external forcing as far as possible, the external forcing is fixed into the lowest two wave number shells, and their specific expressions are shown in the Appendix. Due to the shell limitation of external forcing, the external forcing terms are neglected in the following statistical analysis of kinetic energy and helicity in small scales. Similar external forcing which involves the linear combination of velocity and vorticity field is widely adopted to obtain helical turbulence in the area of incompressible flows [41].

In addition, total energy per unit volume $\mathscr{E}$ is

$$
\mathscr{E}=\frac{p}{\gamma-1}+\frac{1}{2} \rho u_{i} u_{i}
$$

and the viscous stress is

$$
\sigma_{i j}=\mu\left(\frac{\partial u_{i}}{\partial x_{j}}+\frac{\partial u_{j}}{\partial x_{i}}\right)-\frac{2}{3} \mu \frac{\partial u_{k}}{\partial x_{k}} \delta_{i j} .
$$

The viscosity coefficient $\mu$ and thermal conductivity coefficient $\kappa$ are determined by the Sutherland law [42] for simplicity:

$$
\mu=\frac{1.4042 T^{1.5}}{T+0.40417}, \quad \kappa=\frac{1.4042 T^{1.5}}{T+0.40417} .
$$

We performed DNSs of compressible helical turbulence in a cubic box with a length of $2 \pi$. The grid resolution is $1024^{3}$, and the boundary conditions of the three directions are periodic. To obtain high-order numerical results, we adopted a hybrid spatial numerical scheme [43] that adopts an eighth-order compact finite difference scheme [44] in smooth regions and a seventh-order weighted essential nonoscillatory scheme [45] in shock regions, and an explicit low storage second-order Runge-Kutta technique as the time marching scheme [43]. In the lowest two wave number shells, fixed injection rates of kinetic energy and helicity were applied to get a stationary flow field. The thermal cooling function $\Lambda$ was chosen with a uniform expression [46] of $\Lambda=\sigma_{I} T^{0}$, and we set the value of $\sigma_{I}$ to be one in our numerical simulations. Previous studies have confirmed that there is no distinct difference with the different thermal cooling functions [43]. 
TABLE I. Characteristic parameters of steady numerical simulations.

\begin{tabular}{lcccccccc}
\hline \hline Resolution & $\mathrm{Re}_{\lambda}$ & $M_{t}$ & $\mathrm{E}$ & $\mathrm{H}$ & $k_{\max } \eta$ & $\eta / \Delta x$ & $L_{f} / \eta$ & $\lambda / \eta$ \\
\hline $1024^{3}$ & 232 & 0.71 & 2.56 & -2.36 & 3.01 & 0.96 & 205 & 30 \\
\hline \hline
\end{tabular}

Some parameters in isotropic turbulence were employed to characterize the flow field [43,47]. There were some characteristic length scales: the integral length scale $L_{f}$, the Taylor microscale $\lambda$, and the Kolmogorov length scale $\eta$. The definitions of them are

$$
\lambda=\frac{L_{f}=\frac{3 \pi}{2\left(u^{\prime}\right)^{2}} \int_{0}^{\infty} \frac{E(k)}{k} d k,}{\left\langle\left[\left(\partial u_{1} / \partial x_{1}\right)^{2}+\left(\partial u_{2} / \partial x_{2}\right)^{2}+\left(\partial u_{3} / \partial x_{3}\right)^{2}\right] / 3\right\rangle^{1 / 2}},
$$

and

$$
\eta=\left[\langle\mu / \operatorname{Re} \rho\rangle^{3} / \varepsilon\right]^{1 / 4} .
$$

Here $E(k)$ is the spectrum of kinetic energy per unit mass, $k$ is the wave number shell, $u^{\prime}$ is the root-mean square $(\mathrm{rms})$ of velocity vector $\mathbf{u}$ which is defined as $u^{\prime}=\sqrt{\left\langle u_{1}^{2}+u_{2}^{2}+u_{3}^{2}\right\rangle / 3}$, and $\varepsilon$ is the ensemble-averaged viscous dissipation rate of kinetic energy per unit volume, which is defined as $\varepsilon=\left\langle\sigma_{i j} S_{i j} / \operatorname{Re}\right\rangle$. Here the strain-rate tensor is $S_{i j}=(1 / 2)\left(\partial u_{i} / \partial x_{j}+\partial u_{j} / \partial x_{i}\right)$. The Taylor microscale Reynolds number $\operatorname{Re}_{\lambda}$ and the turbulent Mach number $M_{t}$ are defined as

$$
\operatorname{Re}_{\lambda}=\frac{u^{\prime} \lambda\langle\rho\rangle}{\langle\mu\rangle}, \quad M_{t}=M \frac{\sqrt{3} u^{\prime}}{\langle\sqrt{T}\rangle} .
$$

The above characteristic parameters in our steady numerical simulation are summarized in Table I. The value of $\eta / \Delta x$ is approaching one, which reflects the fine grid resolution of our numerical simulations, and $\Delta x$ is the grid spacing in each direction. The relative high $M_{t}=$ 0.71 corresponds to strong compressibility, which paves the way for further investigations into compressible turbulent flows contrary to incompressible turbulent flows. Here $k_{\max }$ is the half of the total grid numbers in one direction, $\mathrm{E}$ is the mean kinetic energy, and $\mathrm{H}$ is the mean helicity. All statistical results in this paper were averaged over 10 instantaneous flow fields across one large-eddy turnover time.

The power-law solutions of kinetic energy and helicity, which ignore any intermittency corrections, can be written as

$$
\begin{array}{rlrl}
E(k) \sim C_{E} \varepsilon^{2 / 3} k^{-5 / 3} ; & H(k) & \sim C_{H} \delta \varepsilon^{-1 / 3} k^{-5 / 3}, \\
E^{\mathrm{R}}(k) \sim \frac{1}{2} \varepsilon^{2 / 3} k^{-5 / 3}\left[1+\frac{\beta}{2 k}\left(\frac{\delta}{\varepsilon}\right)\right] ; & E^{\mathrm{L}}(k) \sim \frac{1}{2} \varepsilon^{2 / 3} k^{-5 / 3}\left[1-\frac{\beta}{2 k}\left(\frac{\delta}{\varepsilon}\right)\right], \\
H^{\mathrm{R}}(k) \sim \varepsilon^{2 / 3} k^{-2 / 3}\left[1+\frac{\beta}{2 k}\left(\frac{\delta}{\varepsilon}\right)\right] ; & H^{\mathrm{L}}(k) \sim \varepsilon^{2 / 3} k^{-2 / 3}\left[1-\frac{\beta}{2 k}\left(\frac{\delta}{\varepsilon}\right)\right],
\end{array}
$$

where the superscripts $\mathrm{R}$ and $\mathrm{L}$ denote right and left chirality, respectively, $C_{E}$ and $C_{H}$ are the Kolmogorov constants of kinetic and helicity spectra, $\beta=C_{H} / C_{E}$, and $\varepsilon$ and $\delta$ are the ensembleaveraged viscous dissipation rate of kinetic energy and helicity per unit volume. We show the compensated spectra of all components of kinetic energy and helicity in Fig. 1. The plateaus of left chirality and the total kinetic energy and helicity spectra illustrated in Fig. 1 reflect the full joint turbulence cascade of our numerical simulations with the left-chirality dominance. The discrepancy of the left- and right-chirality energy (helicity) spectra also reflects the characteristics of the broken 

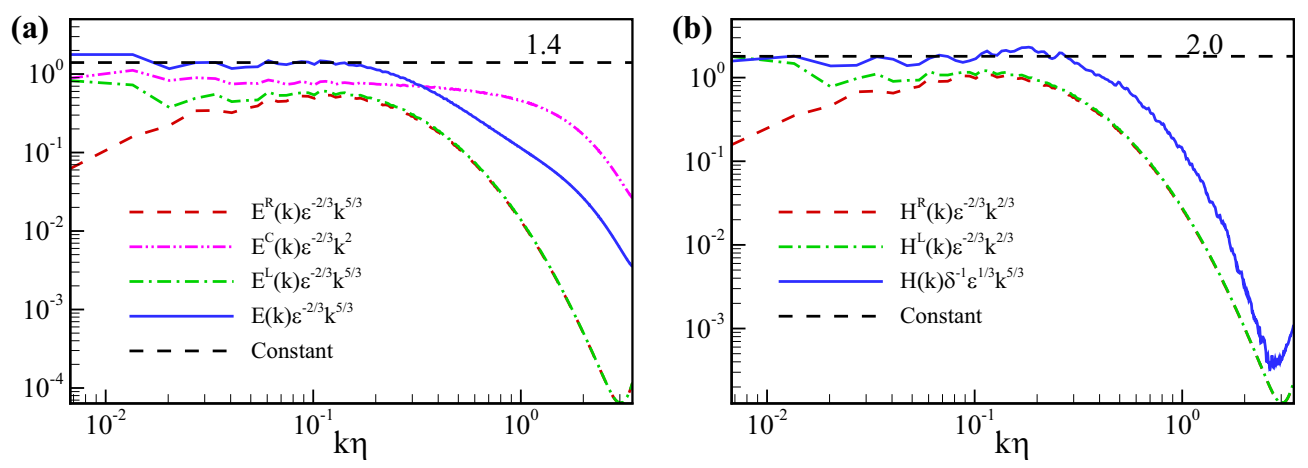

FIG. 1. (a) Compensated spectra of left chirality (L), right chirality (R), free chirality (C), and total kinetic energy. (b) Compensated spectra of left chirality (L), right chirality (R), and total helicity.

mirror symmetry, while the degree of this chirality discrepancy would decrease, with the decrease of length scale. It means that small-scale structures would restore the mirror symmetry statistically. The theory of three-dimensional Burgers turbulence reveals that shock waves lead to a $k^{-2}$ spectrum of the compressible component of velocity [48], and our numerical result in Fig. 1(a) confirms this scaling exponent of the compressible component of velocity. Previous studies suggested that $C_{E}=1.58$ in high-resolution numerical simulations of $\mathrm{Re}_{\lambda} \approx 1000$ [49], and $C_{E}=1.4$ was obtained by eddy-damped quasinormal Markovian (EDQNM) closure [14] and incompressible helical turbulence [50]. Our numerical investigations in Fig. 1(a) revealed that $C_{E}=1.4$, which is lower than our previous result with $C_{E}=1.6$ in highly compressible turbulence [51]. We suppose that the lower value of Kolmogorov constant $C_{E}$ may be attributed to the helicity effect. To be specific, helicity hinders the kinetic energy cascade from large scales to small scales $[32,38,52]$. The reduced kinetic energy in the inertial subrange might also be reflected in the decreased Kolmogorov constant. The other Kolmogorov constant for helicity $C_{H}$ in Fig. 1(b) is approximately 2.0, which is close to the result of $C_{H}=2.23$ obtained by EDQNM closure [14]. It exhibits substantial deviation from the previous conclusions with $C_{H}=1.0$ [50] and the Obukhov-Corrsin constant of a passive scalar spectrum [53-55].

\section{GOVERNING EQUATIONS FOR FILTERED KINETIC ENERGY AND HELICITY}

The evolution equations of homochiral kinetic energy and helicity are prerequisites for further numerically investigating the underlying physical mechanism of the cross-chirality kinetic energy and helicity transfer. In this section, the filtered governing equations of homochiral kinetic energy and helicity are derived, and they describe the evolution characteristics of homochiral kinetic energy and helicity. In compressible turbulence, the filtering method was essentially employed for scale interactions. A good filtering method must satisfy the inviscid criterion, which means that viscous effects on the dynamics of large-scale momentum and kinetic energy could be negligible [28]. Any filtered low-pass variable for primitive variable is defined as

$$
\bar{a}_{l}(\mathbf{x})=\int G_{l}(\mathbf{r}) a(\mathbf{x}+\mathbf{r}) d^{3} \mathbf{r}
$$

where $G_{l}(\mathbf{r})$ is the normalized convolution kernel for various filter types like a top-hat filter, Gaussian filter, and sharp spectral filter. Whichever filter type was chosen, it must be a sufficiently smooth real-valued function and satisfy $\int d^{3} \mathbf{x} G(\mathbf{x})=1$. A top-hat filter is employed to get the 
filtered flow field [56,57], which is homogeneous and defined in one dimension as

$$
\bar{a}_{i}=\frac{1}{4 n}\left(a_{i-n}+2 \sum_{j=i-n+1}^{i+n-1} a_{j}+a_{i+n}\right),
$$

where the filter width $l=2 n \Delta x$, and $\Delta x$ is the grid spacing. The three-dimensional filter is easily obtained through employing the one-dimensional filter in three directions. According to the previous conclusions, there is no distinct discrepancy for some statistical analysis of different filter types [58]. In addition, Favre filtering is a useful filtering method for scale decomposition often used in LES in compressible turbulence. It contains the effect of nonconstant density, and it reads as

$$
\widetilde{a}_{l}=\frac{\overline{(\rho a)}_{l}}{\bar{\rho}_{l}} .
$$

One advantage of Favre filtering is that it provides similar filtered governing equations corresponding to nonfiltered governing equations to explore turbulent cascade [28].

The following filtered governing equations can be easily derived from using the filter method:

$$
\begin{aligned}
\frac{\partial \bar{\rho}}{\partial t}+\frac{\partial\left(\bar{\rho} \tilde{u}_{j}\right)}{\partial x_{j}} & =0 \\
\frac{\partial\left(\bar{\rho} \tilde{u}_{i}\right)}{\partial t}+\frac{\partial\left(\bar{\rho} \tilde{u}_{i} \tilde{u}_{j}\right)}{\partial x_{j}} & =-\frac{\partial \bar{p}}{\partial x_{i}}+\frac{1}{\operatorname{Re}} \frac{\partial \bar{\sigma}_{i j}}{\partial x_{j}}-\frac{\partial \bar{\rho} \tilde{\tau}_{i j}}{\partial x_{j}},
\end{aligned}
$$

where the SGS stress is defined as $\bar{\rho} \widetilde{\tau}_{i j}=\bar{\rho}\left(\widetilde{u_{i} u_{j}}-\widetilde{u}_{i} \widetilde{u}_{j}\right)$. This SGS stress represents the contributions of residual components for the filtered flow field, and this interaction always works in neighboring scales, which corresponds to locality of turbulence cascades [59].

To guarantee the positive definiteness of the kinetic energy spectrum, a density-weighted velocity vector was introduced to study kinetic energy in compressible turbulence [60], and the filtered form is defined as

$$
\widetilde{\mathbf{v}}=\sqrt{\bar{\rho}} \widetilde{\mathbf{u}}
$$

Hence, the large-scale kinetic energy in compressible turbulence can be written as half of the density-weighted velocity squared $|\widetilde{\mathbf{v}}|^{2} / 2$. The governing equation for it reads as

$$
\frac{\partial \widetilde{v}_{i}}{\partial t}+\tilde{u}_{j} \frac{\partial \widetilde{v}_{i}}{\partial x_{j}}+\frac{\widetilde{v}_{i}}{2} \frac{\partial \widetilde{u}_{j}}{\partial x_{j}}=-\frac{1}{\sqrt{\bar{\rho}}} \frac{\partial \bar{p}}{\partial x_{i}}-\frac{1}{\sqrt{\bar{\rho}}} \frac{\partial \bar{\rho} \tilde{\tau}_{i j}}{\partial x_{j}}+\frac{1}{\operatorname{Re}} \frac{1}{\sqrt{\bar{\rho}}} \frac{\partial \bar{\sigma}_{i j}}{\partial x_{j}} .
$$

Helmholtz decomposition was used to divide a vector field in Fourier space into compressible and solenoidal components. In addition, the flow field can further be decomposed into complex helical waves, which provide a pair of orthogonal bases for the divergence-free flow field $[31,61,62]$. These complex helical waves are eigenfunctions of the curl operator, which satisfy

$$
\nabla \times \widetilde{\mathbf{u}}^{\mathrm{R}}=|\mathbf{k}| \widetilde{\mathbf{u}}^{\mathrm{R}}, \quad \nabla \times \widetilde{\mathbf{u}}^{\mathrm{L}}=-|\mathbf{k}| \widetilde{\mathbf{u}}^{\mathrm{L}} .
$$

Similar to Helmholtz decomposition, these eigenfunctions are orthogonal in the Fourier space and functional orthogonal in physical space, which means that

$$
\widetilde{\mathbf{u}}^{\mathrm{R}}(\mathbf{k}) \cdot \widetilde{\mathbf{u}}^{\mathrm{L} *}(\mathbf{k})=0,\left\langle\mathbf{u}^{\mathrm{R}}(\mathbf{x}), \mathbf{u}^{\mathrm{L}}(\mathbf{x})\right\rangle=\int \mathbf{u}^{\mathrm{R}}(\mathbf{x}) \cdot \mathbf{u}^{\mathrm{L} *}(\mathbf{x}) d \mathbf{x}=0,
$$

where $*$ denotes the complex conjugation. In summary, the solenoidal-free velocity field corresponds to a 0 eigenvalue of the curl operation, and we can write the following uniform expression which provides three orthogonal bases for flow field decomposition:

$$
\mathbf{u}(\mathbf{k})=\widetilde{\mathbf{u}}^{\mathrm{R}}(\mathbf{k})+\widetilde{\mathbf{u}}^{\mathrm{L}}(\mathbf{k})+\widetilde{\mathbf{u}}^{\mathrm{C}}(\mathbf{k})
$$


Helmholtz Decomposition

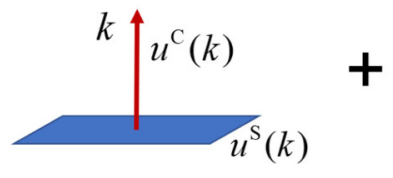

Helical Wave Decomposition

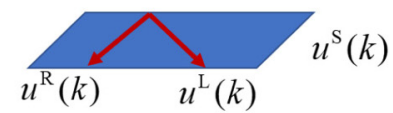

General Helical Wave Decomposition

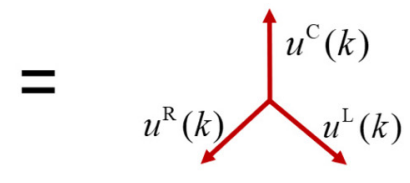

FIG. 2. Sketch of Helmholtz decomposition, helical wave decomposition, and general helical wave decomposition. The superscript $S$ denotes solenoidal component of velocity.

It means that Helmholtz decomposition is a special form of HWD. Hence, we combined the two popular decomposition methods to propose a decomposition for compressible turbulence, named general helical wave decomposition. Figure 2 shows a schematic sketch of velocity relations in different modes of Helmholtz decomposition, HWD and general helical wave decomposition.

Basing on above flow field decomposition method, we can decompose filtered density-weighted velocity $\widetilde{\mathbf{v}}$ as

$$
\widetilde{\mathbf{v}}=\widetilde{\mathbf{v}}^{\mathrm{L}}+\widetilde{\mathbf{v}}^{\mathrm{R}}+\widetilde{\mathbf{v}}^{\mathrm{C}}
$$

Multiplying Eq. (20) by $\widetilde{v}_{i}^{\mathrm{X}}$ yields

$$
\widetilde{v}_{i}^{\mathrm{X}} \frac{\partial \widetilde{v}_{i}}{\partial t}+\widetilde{v}_{i}^{\mathrm{X}}\left(\widetilde{u}_{j} \frac{\partial \widetilde{v}_{i}}{\partial x_{j}}+\frac{\widetilde{v}_{i}}{2} \frac{\partial \widetilde{u}_{j}}{\partial x_{j}}\right)=-\frac{\widetilde{v}_{i}^{\mathrm{X}}}{\sqrt{\bar{\rho}}} \frac{\partial \bar{p}}{\partial x_{i}}-\frac{\widetilde{v}_{i}^{\mathrm{X}}}{\sqrt{\bar{\rho}}} \frac{\partial \bar{\rho} \widetilde{\tau}_{i j}}{\partial x_{j}}+\frac{1}{\operatorname{Re}} \frac{\widetilde{v}_{i}^{\mathrm{X}}}{\sqrt{\bar{\rho}}} \frac{\partial \bar{\sigma}_{i j}}{\partial x_{j}} .
$$

where $\mathrm{X}=\mathrm{L}, \mathrm{C}, \mathrm{R}$ correspond to left-chirality, free-chirality (compressible), and right-chirality components of filtered density-weighted velocity $\widetilde{\mathbf{v}}$. Making an ensemble average of the above equation yields the following filtered governing equations for these three components:

$$
\frac{\partial}{\partial t}\left\langle\frac{1}{2}\left(\widetilde{v}_{i}^{\mathrm{X}}\right)^{2}\right\rangle=\left\langle\mathrm{A}_{l}^{\mathrm{EX}}\right\rangle-\left\langle\Phi_{l}^{\mathrm{EX}}\right\rangle-\left\langle\Pi_{l}^{\mathrm{EX}}\right\rangle-\left\langle\mathrm{D}_{l}^{\mathrm{EX}}\right\rangle
$$

where the nonlinear advection term $\mathrm{A}_{l}^{\mathrm{EX}}$, the pressure-dilatation term $\Phi_{l}^{\mathrm{EX}}$, the kinetic energy flux $\Pi_{l}^{\mathrm{EX}}$, and the viscous dissipation term $\mathrm{D}_{l}^{\mathrm{EX}}$ are defined as

$$
\begin{gathered}
\mathrm{A}_{l}^{\mathrm{EX}}=-\widetilde{v}_{i}^{\mathrm{X}}\left(\tilde{u}_{j} \frac{\partial \widetilde{v}_{i}}{\partial x_{j}}+\frac{\widetilde{v}_{i}}{2} \frac{\partial \widetilde{u}_{j}}{\partial x_{j}}\right), \\
\Phi_{l}^{\mathrm{EX}}=-\bar{p} \frac{\partial}{\partial x_{i}}\left(\frac{\widetilde{v}_{i}^{\mathrm{X}}}{\sqrt{\bar{\rho}}}\right), \\
\Pi_{l}^{\mathrm{EX}}=-\bar{\rho} \widetilde{\tau}_{i j} \frac{\partial}{\partial x_{j}}\left(\frac{\widetilde{v}_{i}^{\mathrm{X}}}{\sqrt{\bar{\rho}}}\right),
\end{gathered}
$$

and

$$
\mathrm{D}_{l}^{\mathrm{EX}}=\frac{\bar{\sigma}_{i j}}{\operatorname{Re}} \frac{\partial}{\partial x_{j}}\left(\frac{\widetilde{v}_{i}^{\mathrm{X}}}{\sqrt{\bar{\rho}}}\right) .
$$

The above governing equations determine the space-time evolutions of homochiral kinetic energy. The nonlinear advection term $\mathrm{A}_{l}^{\mathrm{EX}}$ involves the spatial transport of homochiral kinetic energy, and the couplings with other two homochiral modes of kinetic energy. The pressure-dilatation term $\Phi_{l}^{\mathrm{EX}}$ involves the correlations of pressure field and velocity field, and the main differences differing from incompressible flows are reflected on the variable density and non-divergence-free velocity. The kinetic energy flux $\Pi_{l}^{\mathrm{EX}}$ denotes the cross-scale homochiral kinetic energy transfer statistically. The last viscous dissipation term $\mathrm{D}_{l}^{\mathrm{EX}}$ describes the transfer of homochiral kinetic energy to internal energy. The various statistical characteristics of the nonlinear advection term involving the cross-chirality transfer will be discussed in detail in the next section. 
Next, we show the detail derivation of the governing equations of homochiral helicity in compressible flows. Expanding and dividing Eq. (18b) by $\bar{\rho}$ yield

$$
\frac{\partial \tilde{u}_{i}}{\partial t}+\tilde{u}_{j} \frac{\partial \tilde{u}_{i}}{\partial x_{j}}=-\frac{1}{\bar{\rho}} \frac{\partial \bar{p}}{\partial x_{i}}+\frac{1}{\operatorname{Re}} \frac{1}{\bar{\rho}} \frac{\partial \bar{\sigma}_{i j}}{\partial x_{j}}-\frac{1}{\bar{\rho}} \frac{\partial \bar{\rho} \tilde{\tau}_{i j}}{\partial x_{j}} .
$$

Making a curl operation of Eq. (27), and we can get the governing equation of filtered vorticity vector $\omega$ as

$$
\frac{\partial \widetilde{\omega}_{i}}{\partial t}+\widetilde{u}_{j} \frac{\partial \widetilde{\omega}_{i}}{\partial x_{j}}-\widetilde{\omega}_{j} \frac{\partial \widetilde{u}_{i}}{\partial x_{j}}+\widetilde{\omega}_{i} \frac{\partial \widetilde{u}_{j}}{\partial x_{j}}=\frac{1}{\bar{\rho}^{2}}(\nabla \bar{\rho} \times \nabla \bar{p})_{i}+\frac{1}{\operatorname{Re}} \nabla \times\left(\frac{1}{\bar{\rho}} \frac{\partial \bar{\sigma}_{i j}}{\partial x_{j}}\right)-\nabla \times\left(\frac{1}{\bar{\rho}} \frac{\partial \bar{\rho} \widetilde{\tau}_{i j}}{\partial x_{j}}\right) .
$$

Therefore, we get the following governing equation of filtered X-component helicity in compressible turbulence,

$$
\begin{aligned}
& \frac{\partial\left(\widetilde{u}_{i}^{\mathrm{X}} \widetilde{\omega}_{i}^{\mathrm{X}}\right)}{\partial t}+\widetilde{\omega}_{i}^{\mathrm{X}} \widetilde{u}_{j} \frac{\partial \widetilde{u}_{i}}{\partial x_{j}}+\widetilde{u}_{i}^{\mathrm{X}} \frac{\partial\left(\omega_{i} u_{j}-\omega_{j} u_{i}\right)}{\partial x_{j}} \\
& =-\frac{\widetilde{\omega}_{i}^{\mathrm{X}}}{\bar{\rho}} \frac{\partial \bar{p}}{\partial x_{i}}+\frac{\widetilde{u}_{i}^{\mathrm{X}}}{\bar{\rho}^{2}}(\nabla \bar{\rho} \times \nabla \bar{p})_{i}+\frac{\widetilde{\omega}_{i}^{\mathrm{X}}}{\operatorname{Re}} \frac{1}{\bar{\rho}} \frac{\partial \bar{\sigma}_{i j}}{\partial x_{j}}+\frac{\widetilde{u}_{i}^{\mathrm{X}}}{\operatorname{Re}} \nabla \times\left(\frac{1}{\bar{\rho}} \frac{\partial \bar{\sigma}_{i j}}{\partial x_{j}}\right) \\
& \quad-\frac{\widetilde{\omega}_{i}^{\mathrm{X}}}{\bar{\rho}} \frac{\partial \bar{\rho} \widetilde{\tau}_{i j}}{\partial x_{j}}-\widetilde{u}_{i}^{\mathrm{X}} \nabla \times\left(\frac{1}{\bar{\rho}} \frac{\partial \bar{\rho} \widetilde{\tau}_{i j}}{\partial x_{j}}\right) .
\end{aligned}
$$

Making an ensemble average of the above equation, we get the governing equation of filtered ensemble-average $\mathrm{X}$-component helicity as

$$
\frac{\partial \widetilde{H}_{l}^{\mathrm{X}}}{\partial t}=\left\langle\mathrm{A}_{l}^{\mathrm{HX}}\right\rangle-\left\langle\Phi_{l}^{\mathrm{HX}}\right\rangle-\left\langle\Pi_{l}^{\mathrm{HX}}\right\rangle-\left\langle\mathrm{D}_{l}^{\mathrm{HX}}\right\rangle,
$$

where the nonlinear advection term $\mathrm{A}_{l}^{\mathrm{HX}}$, pressure term $\Phi_{l}^{\mathrm{HX}}$, helicity flux $\Pi_{l}^{\mathrm{HX}}$, and viscous dissipation term $\mathrm{D}_{l}^{\mathrm{HX}}$ are defined as

$$
\begin{gathered}
\mathrm{A}_{l}^{\mathrm{HX}}=-2 \widetilde{\omega}_{i}^{\mathrm{X}} \widetilde{u}_{j} \frac{\partial \widetilde{u}_{i}}{\partial x_{j}}, \\
\Phi_{l}^{\mathrm{HX}}=2 \bar{p} \frac{\partial}{\partial x_{i}}\left(\frac{\widetilde{\omega}_{i}^{\mathrm{X}}}{\bar{\rho}}\right), \\
\Pi_{l}^{\mathrm{HX}}=2 \bar{\rho} \widetilde{\tau}_{i j} \frac{\partial}{\partial x_{j}}\left(\frac{\widetilde{\omega}_{i}^{\mathrm{X}}}{\bar{\rho}}\right),
\end{gathered}
$$

and

$$
\mathrm{D}_{l}^{\mathrm{HX}}=\frac{2 \bar{\sigma}_{i j}}{\operatorname{Re}} \frac{\partial}{\partial x_{j}}\left(\frac{\widetilde{\omega}_{i}^{\mathrm{X}}}{\bar{\rho}}\right) .
$$

Similarly, the nonlinear advection term $\mathrm{A}_{l}^{\mathrm{HX}}$ not only describes the homochiral spatial transport of helicity, but also involves the couplings of the other-mode helicity. Contrasted to the incompressible issue, the complicated nonlinear term involves the effect of the compressive component of velocity. Also, the pressure term $\Phi_{l}^{\mathrm{HX}}$ is at present obviously in the governing equation of helicity. Nevertheless, this term would be equal to zero when the density is constant in incompressible flows. Contrasted to the pressure-dilatation term in the governing equation of kinetic energy (26a), this pressure term $\Phi_{l}^{\mathrm{HX}}$ gets rid of the effect of velocity divergence and is related to the variable density. More details were explored in our previous work [30]. The homochiral helicity flux $\Pi_{l}^{\mathrm{HX}}$ denotes the cross-scale helicity transfer, and it is related to the chirality of vorticity. Although the definition of helicity in compressible flows is the same as that in incompressible flows, the compressive component of velocity takes a part in the dynamical evolutions of total and homochiral helicity. Hence, the effect of compressibility on the dynamical evolutions of helicity would result in a more 


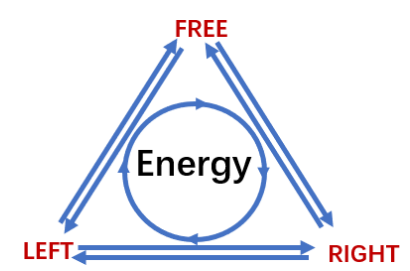

FIG. 3. Sketch of kinetic energy transfer among left-, right-, and free-chirality modes.

complicated helicity transfer process. The further statistical characteristics of the above involved terms will be investigated numerically in detail in the next section.

\section{STATISTICAL ANALYSIS OF THE CROSS-CHIRALITY KINETIC ENERGY AND HELICITY TRANSFER}

In this section, the cross-chirality kinetic energy and helicity transfer functions are further decomposed, according to the involved chiral velocity and vorticity in their definitions. The methodology refers to a previous valuable literature [34]. We select the dominant chiral mode (i.e., the free-chirality mode for kinetic energy and the left-chirality mode for helicity) to investigate the regulations of cross-chirality transfer, especially under the influence of compressibility. The effects of compression and expansion on the chirality transfers of kinetic energy and helicity reveal the underlying mechanism of turbulent systems and provide some theoretical foundations to predict the local characteristics of chirality transfer in general compressible turbulent flows.

\section{A. The kinetic energy transfer among left, right, and free chirality}

Besides the kinetic energy cascade procedure with scale interactions, there is another mechanism for homochiral kinetic energy transfer. This mechanism originates from nonlinear interactions among different chiralities in the same length scales, which appears in the filtered kinetic energy governing equation. The two dominant chiral modes (i.e., free and left chirality) are selected to investigate the cross-chirality kinetic energy transfer. The cross-chirality nonlinear term of free-chirality kinetic energy governing equation reads as

$$
\mathrm{A}_{l}^{\mathrm{CMN}}=-\widetilde{v}_{i}^{\mathrm{C}}\left(\widetilde{u}_{j}^{\mathrm{M}} \frac{\partial \widetilde{v}_{i}^{\mathrm{N}}}{\partial x_{j}}+\frac{\widetilde{v}_{i}^{\mathrm{N}}}{2} \frac{\partial \widetilde{u}_{j}^{\mathrm{M}}}{\partial x_{j}}\right), \quad \mathrm{M}, \mathrm{N}=\mathrm{L}, \mathrm{R}, \mathrm{C} .
$$

The cross-chirality nonlinear term of the left-chirality kinetic energy governing equation reads as

$$
\mathrm{A}_{l}^{\mathrm{LMN}}=-\widetilde{v}_{i}^{\mathrm{L}}\left(\widetilde{u}_{j}^{\mathrm{M}} \frac{\partial \widetilde{v}_{i}^{\mathrm{N}}}{\partial x_{j}}+\frac{\widetilde{v}_{i}^{\mathrm{N}}}{2} \frac{\partial \widetilde{u}_{j}^{\mathrm{M}}}{\partial x_{j}}\right), \quad \mathrm{M}, \mathrm{N}=\mathrm{L}, \mathrm{R}, \mathrm{C} .
$$

Here the superscripts L, R, C denote the left-, right-, and free-chirality mode, respectively. From the definition of nonlinear interactions, it can be divided into three categories, and the three categories denote three kinetic energy transfer routes from other chiral modes. For the free-chirality kinetic energy, it receives kinetic energy from the left-chirality mode, marked with $\mathrm{A}_{l}^{\mathrm{CL}}$, from the rightchirality mode $\mathrm{A}_{l}^{\mathrm{CR}}$, and they can be denoted as the pairwise interaction. The third transfer routine is from both the left- and right-chirality mode $\mathrm{A}_{l}^{\mathrm{CLR}}$, and it can be denoted as the triple interaction in the following. This classification method applies equally to the nonlinear interactions of leftchirality kinetic energy, and it depends on the specific selections of symbols M and N. These three categories are illustrated schematically in Fig. 3.

The ensemble averages of left- and free-chirality modes receiving from other chiral modes are exhibited in Fig. 4. It is worthwhile reminding here that the strength orders of all-chirality modes from high to low are free-, left-, and right-chirality modes. For free-chirality kinetic energy 

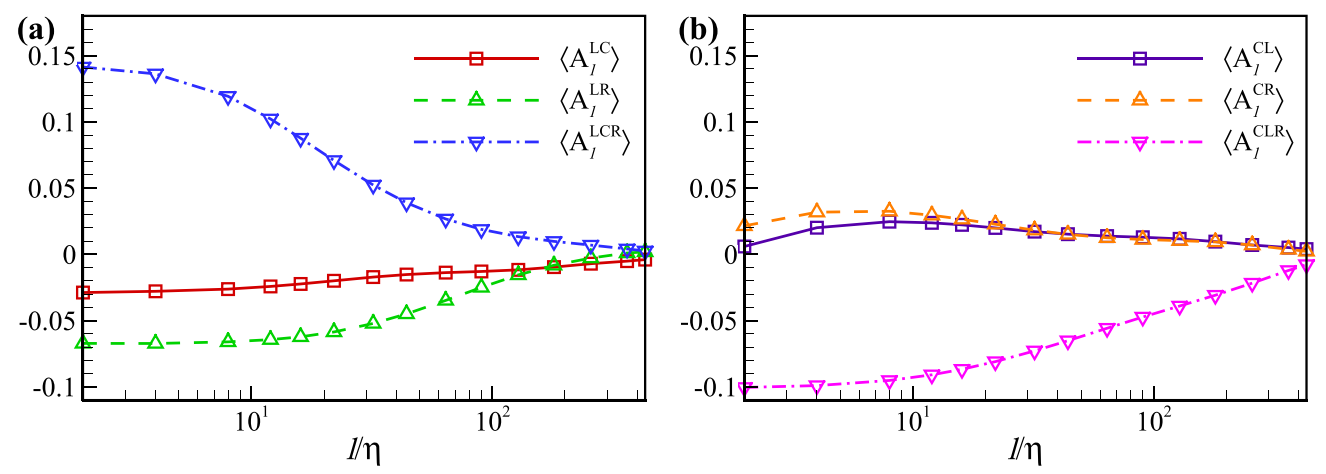

FIG. 4. (a) Ensemble averages of left-chirality kinetic energy receiving from free-chirality mode $\mathrm{A}_{l}^{\mathrm{LC}}$, receiving from right-chirality mode $\mathrm{A}_{l}^{\mathrm{LR}}$, and receiving from free- and right-chirality mode $\mathrm{A}_{l}^{\mathrm{LCR}}$. (b) Ensemble averages of free-chirality kinetic energy receiving from left-chirality mode $\mathrm{A}_{l}^{\mathrm{CL}}$, receiving from right-chirality mode $\mathrm{A}_{l}^{\mathrm{CR}}$, and receiving from left- and right-chirality mode $\mathrm{A}_{l}^{\mathrm{CLR}}$.

as the strongest chiral mode, it transfers kinetic energy to other chiral modes through nonlinear interactions on the whole. The numerical consequences in Fig. 4(b) show that the amplitude of the triple interaction is the largest, and its negative sign is consistent with the total transfer regulations. Nevertheless, the pairwise interaction is secondary, and its positive sign corresponds to an inverse transfer of kinetic energy relative to triple interaction. For left-chirality kinetic energy as a secondary chiral mode, we infer that it should receive kinetic energy through nonlinear interactions on the whole. The numerical results shown in Fig. 4(a) reveal that the triple interaction is also dominant, relative to the pairwise interaction. The amplitude of the triple interaction is the largest, and its sign is positive. It means that left chirality receives kinetic energy from the other two chiral modes through triple interaction. However, the amplitude of the pairwise interaction is smaller, and its negative sign means that it transfers kinetic energy to other chiral modes. Therefore, we can conclude that the triple interaction involving all chiral modes dominates the chirality transfer of kinetic energy totally, while the pairwise interaction is secondary and makes an inverse contribution. The dominant status of triple interactions highlights the complexity of compressible helical turbulence, in contrast to previous incompressible helical turbulence.

In addition, with the decrease of length scales, the amplitudes of all kinetic energy transfer routes increase gradually, and they are comparable to homochiral kinetic energy flux at relatively large scales. At small length scales, the chirality transfer of kinetic energy is stronger than crossscale kinetic energy transfer, and it serves as a complex physical mechanism along with viscous dissipation. The local transfer of kinetic energy at small scales is very strong relative to transfer on the whole [63], and our numerical results show that the chirality transfer of kinetic energy at small scales is also strong. The above statistical regulations of kinetic energy transfer at small scales reveal the complex characteristics of turbulent motions, besides the important viscous dissipation traditionally.

To study the effects of compression and expansion on the chirality transfer of kinetic energy between left and right chiralities, we made the conditional ensemble averages of the cross-chirality transfer function $\mathrm{A}_{l}^{\mathrm{LR}}$ in compression and expansion regions in Fig. 5(a) and their ratios in Fig. 5(b). The numerical results in compression regions are consistent with the regulations exhibited in Fig. 4(a), while the expansion makes an opposite contribution for left-chirality transfer on the whole. Although the amplitudes of the ensemble averages of $\mathrm{A}_{l}^{\mathrm{LR}}$ in expansion regions are smaller than those in compression regions, the expansion of fluid elements can result in an inverse transfer of kinetic energy and lead to a stronger lack of mirror symmetry via pairwise interaction $\mathrm{A}_{l}^{\mathrm{LR}}$. With the decrease of length scales, the ratio of the absolute values of the ensemble averages of 

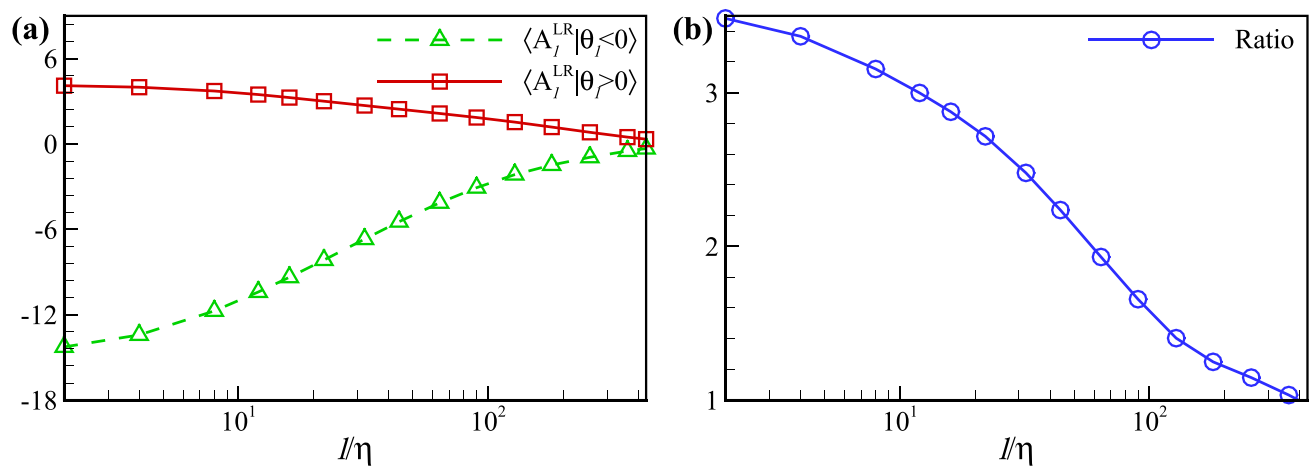

FIG. 5. (a) Conditional ensemble averages of kinetic energy transfer function between left and right chirality $\mathrm{A}_{l}^{\mathrm{LR}}$ in compression regions and in expansion regions. (b) The ratio of the absolute values of the conditional ensemble averages of $\mathrm{A}_{l}^{\mathrm{LR}}$ in compression regions and in expansion regions.

$\mathrm{A}_{l}^{\mathrm{LR}}$ in compression and expansion regions gradually increases, which is related to the dominant compression regions in small scales in our numerical simulations.

Similarly, we made the conditional ensemble averages of cross-chirality kinetic energy transfer functions $\mathrm{A}_{l}^{\mathrm{CL}}, \mathrm{A}_{l}^{\mathrm{CR}}$, and $\mathrm{A}_{l}^{\mathrm{CLR}}$ in compression and expansion regions in Fig. 6(a) and their ratios in compression and expansion regions in Fig. 6(b). For free-chirality kinetic energy, the numerical results of all cross-chirality kinetic energy transfer functions in compression regions are consistent with the results in Fig. 4(a), which reflects a dominant role of compressibility in our numerical simulations. However, the numerical results of the transfer function in expansion regions are also completely different from total trends. The ratio curves of the amplitudes of the ensemble averages of $\mathrm{A}_{l}^{\mathrm{CL}}$ and $\mathrm{A}_{l}^{\mathrm{CR}}$ in compression and expansion regions almost overlap. It means that there exists a negligible difference for left- and right-chirality statistical properties. Conversely, the leading triple interaction is less sensitive to compressibility. Hence, we could conclude that expansion can lead to a stronger lack of mirror symmetry.

\section{B. The helicity transfer among left, right, and free chirality}

There exists another mechanism for helicity transfer in the same length scales, besides the crossscale helicity cascade. We rewrite the Eqs. (30b) as follows and make an identical transformation:
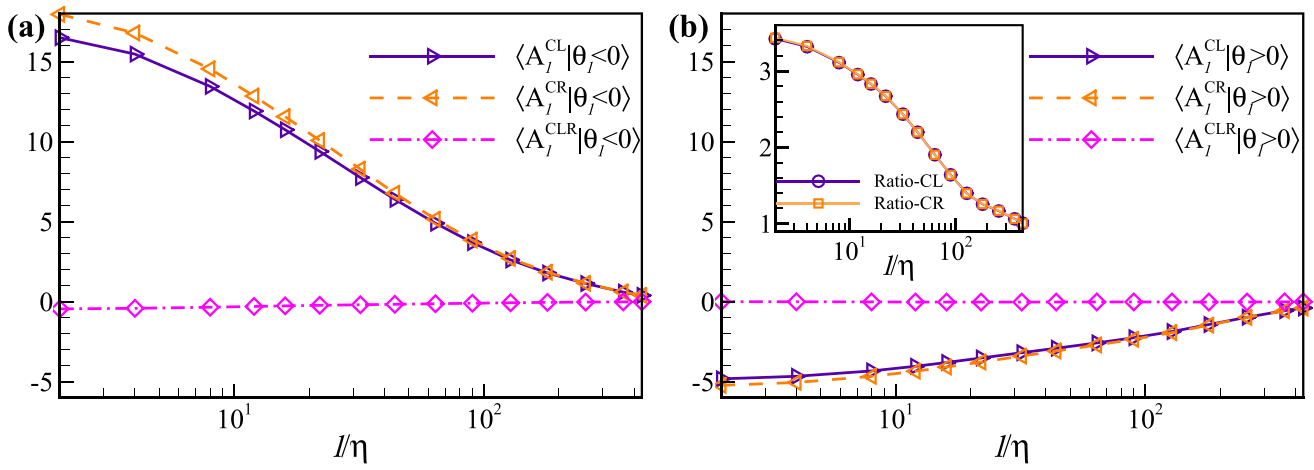

FIG. 6. Conditional ensemble averages of kinetic energy transfer functions $\mathrm{A}_{l}^{\mathrm{CL}}, \mathrm{A}_{l}^{\mathrm{CR}}$, and $\mathrm{A}_{l}^{\mathrm{CLR}}$ in compression regions (a) and in expansion regions (b). The inset is for their ratios in compression and expansion regions. Ratio-CL corresponds to $\mathrm{A}_{l}^{\mathrm{CL}}$, and Ratio-CR corresponds to $\mathrm{A}_{l}^{\mathrm{CR}}$. 


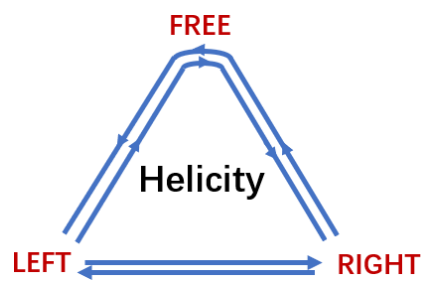

FIG. 7. Sketch of helicity transfer between left- and right-chirality modes.

$$
\mathrm{A}_{l}^{\mathrm{HX}}=-2 \widetilde{\omega}_{i}^{\mathrm{X}} \widetilde{u}_{j} \frac{\partial \widetilde{u}_{i}}{\partial x_{j}}=-2 \widetilde{\boldsymbol{\omega}}^{\mathrm{X}} \cdot\left[\widetilde{\boldsymbol{\omega}} \times \widetilde{\mathbf{u}}+\nabla\left(\frac{1}{2} \widetilde{\mathbf{u}}^{2}\right)\right] .
$$

If we make an ensemble average of the above equation, the following reduced identity could be deduced by homogeneity condition,

$$
\left\langle\mathrm{A}_{l}^{\mathrm{HX}}\right\rangle=-2\left\langle\widetilde{\boldsymbol{\omega}}^{\mathrm{X}} \cdot(\widetilde{\boldsymbol{\omega}} \times \widetilde{\mathbf{u}})\right\rangle .
$$

Surprisingly, the above expression for compressible turbulence is the same to that for incompressible turbulence. However, the only discrepancy lies on the velocity, especially the compressible component. Generally, the nonlinear advection term among different chiralities on the same length scales can be rewritten as

$$
\left\langle\mathrm{H}_{l}^{\mathrm{OMN}}\right\rangle=-2\left\langle\widetilde{\boldsymbol{\omega}}^{\mathrm{O}} \cdot\left(\widetilde{\boldsymbol{\omega}}^{\mathrm{M}} \times \widetilde{\mathbf{u}}^{\mathrm{N}}\right)\right\rangle, \quad \mathrm{O}, \mathrm{M}=\mathrm{L}, \mathrm{R}, \quad \mathrm{N}=\mathrm{L}, \mathrm{C}, \mathrm{R} .
$$

The conservation condition $\left(\left\langle\mathrm{H}_{l}^{\mathrm{OMN}}\right\rangle=0\right)$ can be easily satisfied when the two vorticities are in the same chirality, namely, $\mathrm{O}=\mathrm{M}$. So all components of interactions among different chiralities for the left-chirality helicity can be expressed as

$$
\left\langle\mathrm{H}_{l}^{\mathrm{LRX}}\right\rangle=-2\left[\left\langle\widetilde{\boldsymbol{\omega}}^{\mathrm{L}} \cdot\left(\widetilde{\boldsymbol{\omega}}^{\mathrm{R}} \times \widetilde{\mathbf{u}}^{\mathrm{L}}\right)\right\rangle+\left\langle\widetilde{\boldsymbol{\omega}}^{\mathrm{L}} \cdot\left(\widetilde{\boldsymbol{\omega}}^{\mathrm{R}} \times \widetilde{\mathbf{u}}^{\mathrm{R}}\right)\right\rangle+\left\langle\widetilde{\boldsymbol{\omega}}^{\mathrm{L}} \cdot\left(\widetilde{\boldsymbol{\omega}}^{\mathrm{R}} \times \widetilde{\mathbf{u}}^{\mathrm{C}}\right)\right\rangle\right] .
$$

The third term on the right-hand side of Eq. (36) is present only in compressible flows, and the above three terms can be classified as

$$
\left\langle\mathrm{H}_{l}^{\mathrm{LR}}\right\rangle=-2\left[\left\langle\widetilde{\boldsymbol{\omega}}^{\mathrm{L}} \cdot\left(\widetilde{\boldsymbol{\omega}}^{\mathrm{R}} \times \widetilde{\mathbf{u}}^{\mathrm{L}}\right)\right\rangle+\left\langle\widetilde{\boldsymbol{\omega}}^{\mathrm{L}} \cdot\left(\widetilde{\boldsymbol{\omega}}^{\mathrm{R}} \times \widetilde{\mathbf{u}}^{\mathrm{R}}\right)\right\rangle\right],
$$

which reflects the contributions for left-chirality helicity from the interactions of left- and rightchirality velocity and vorticity. Also,

$$
\left\langle\mathrm{H}_{l}^{\mathrm{LRC}}\right\rangle=-2\left[\left\langle\widetilde{\boldsymbol{\omega}}^{\mathrm{L}} \cdot\left(\widetilde{\boldsymbol{\omega}}^{\mathrm{R}} \times \widetilde{\mathbf{u}}^{\mathrm{C}}\right)\right\rangle\right],
$$

which reflects the contributions for the left-chirality helicity from the interactions of left-, right-, and free-chirality velocity and vorticity. Hence, we can conclude that the compressible component of velocity plays a role of medium for helicity transfer among different chiralities in compressible turbulence, which is the only feature relative to incompressible turbulence. The helicity transfer procedure is illustrated schematically in Fig. 7.

The ensemble average and RMS values of helicity transfer among chiral modes $\left(\mathrm{H}_{l}^{\mathrm{LR}}, \mathrm{H}_{l}^{\mathrm{LRC}}\right)$ are exhibited in Fig. 8. The statistical results reveal that the helicity transfer at small scales is stronger than that at large scales of either $\mathrm{H}_{l}^{\mathrm{LR}}$ or $\mathrm{H}_{l}^{\mathrm{LRC}}$, which should not be ignored particularly at small scales. In addition, as a medium, the compressible component of velocity leads to a weaker helicity transfer, even though it is stronger than the solenoidal component of velocity in our numerical simulations. The fluctuation of helicity transfer via $\mathrm{H}_{l}^{\mathrm{LRC}}$ is stronger because of the compressible component, which can be attributed to the randomly distributed shocklets.

The statistical results of $\mathrm{H}_{l}^{\mathrm{LR}}$ and $\mathrm{H}_{l}^{\mathrm{LRC}}$ in compression, expansion, straining, and rotating regions are shown in Fig. 9. The compression and expansion procedure of fluid elements correspond to two 

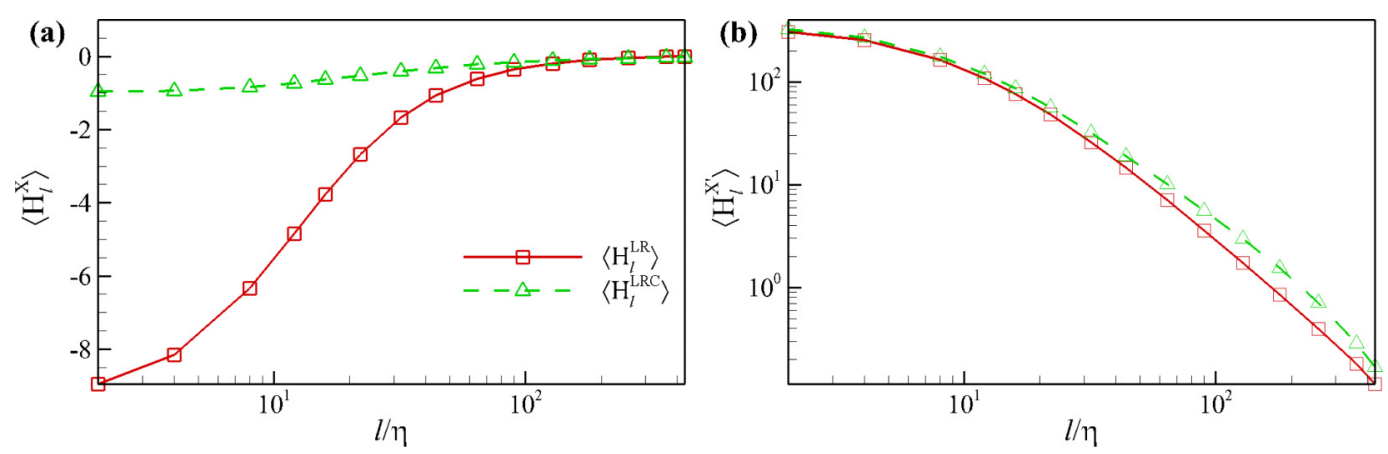

FIG. 8. Ensemble average (a) and root-mean-square (RMS) values (b) of $\mathrm{H}_{l}^{\mathrm{LR}}, \mathrm{H}_{l}^{\mathrm{LRC}}$.

opposite contributions for helicity transfer, via $\mathrm{H}_{l}^{\mathrm{LRC}}$, and this is the only way for a statistical inverse transfer of helicity among different chiral modes. Nevertheless, the absolute value of the ratio is less than one, which means that as an underlying mechanism the inverse transfer of helicity in compression regions is not dominant. The compression procedure is dominant via $\mathrm{H}_{l}^{\mathrm{LR}}$, particularly at small length scales. There is virtually no discrepancy of the influence of rotating and straining procedure on the helicity transfer via $\mathrm{H}_{l}^{\mathrm{LRC}}$. The rotating procedure is dominant for the helicity transfer via $\mathrm{H}_{l}^{\mathrm{LR}}$ for nearly all the length scales. Hence, we can conclude that the rotating procedure contributes mostly to helicity transfer among different chiral modes via $\mathrm{H}_{l}^{\mathrm{LR}}$ even in highly compressible turbulence. The effect of compressibility reflected by $\mathrm{H}_{l}^{\mathrm{LRC}}$ usually works in high compression or expansion regions. The compressibility leads to an inverse transformation of helicity via $\mathrm{H}_{l}^{\mathrm{LRC}}$, and it strengthens the lack of mirror symmetry. However, the effect of compressibility on the dominant transfer routine is consistent with the total transfer regulation. Hence, we infer that the local inverse transfer of helicity is associated with the compressible component of velocity in compression regions via $\mathrm{H}_{l}^{\mathrm{LRC}}$.

\section{CONCLUSIONS}

In this paper, we have investigated the cross-chirality kinetic energy and helicity transfer through carrying out direct numerical simulations of compressible helical and isotropic turbulence at the grid resolution of $1024^{3}$. We focus on the cross-chirality kinetic energy and helicity transfer,
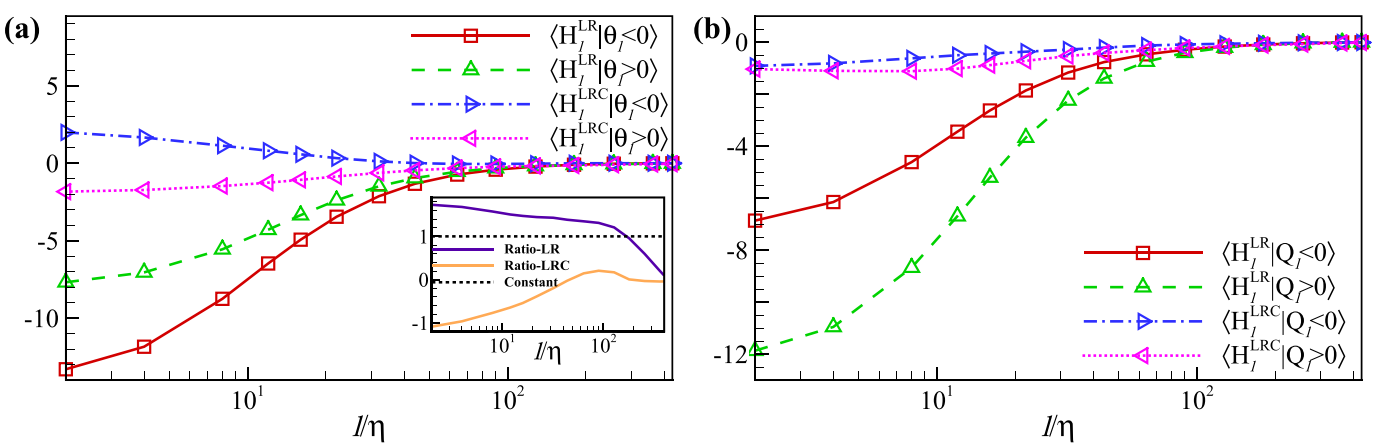

FIG. 9. Conditional ensemble average of $\mathrm{H}_{l}^{\mathrm{LR}}, \mathrm{H}_{l}^{\mathrm{LRC}}$ in compression regions $\left(\theta_{l}<0\right)$ and expansion regions $\left(\theta_{l}>0\right)(\mathrm{a})$, in straining regions $\left(Q_{l}<0\right)$ and rotating regions $\left(Q_{l}>0\right)(\mathrm{b})$. The ratios of the ensemble averages of $\mathrm{H}_{l}^{\mathrm{LR}}, \mathrm{H}_{l}^{\mathrm{LRC}}$ in compression and expansion regions are shown in the inset. Ratio-LR corresponds to $\mathrm{H}_{l}^{\mathrm{LR}}$, and Ratio-LRC corresponds to $\mathrm{H}_{l}^{\mathrm{LRC}}$. 
particularly under the influence of the compression and expansion, and the straining and rotating of fluid elements. We propose a decomposition method of compressible helical turbulence named general helical wave decomposition to investigate the statistical properties of the cross-chirality kinetic energy and helicity transfer in the same length scale.

We conclude that the triple interactions of the left, free, and right chirality dominate the crosschirality kinetic energy transfer, especially at small scales. It is regarded as a physical mechanism for homochiral kinetic energy transfer, which is comparable with kinetic energy flux at relatively large scales and with viscous dissipation at relatively small scales. However, pairwise interaction is secondary, and it is very sensitive to compressibility. Under the influence of expansion, kinetic energy transfers in reverse to stronger chiral modes via pairwise interaction, leading to a stronger lack of mirror symmetry. Helicity transfer among different chiral modes in compressible flows is very similar to that in incompressible flows, and the only discrepancy lies on the medium role of the compressible component of velocity. Although the free-chirality kinetic energy is the strongest, the helicity transfer via the compressible component is weak relative to the interactions of left- and right-chirality velocity and vorticity. The only possibility of the inverse direction of helicity transfer occurs in compression regions statistically, and the compressibility leads to a local stronger lack of mirror symmetry via the compressible component of velocity. However, the compressibility also has a drastic influence on the dominant helicity transfer routine, which promotes the total balance of chirality. As an underlying mechanism of chirality transfer of helicity, the statistical results via the compressible component of velocity do not conflict with the total effect of compressibility for helicity transfer.

In summary, the expansion is highlighted to serve as an important role to promote the inverse chirality transfer of kinetic energy statistically, and further strengthens the lack of mirror symmetry. The compression is regarded as a local inverse helicity transfer routine via the compressible component of velocity and further influences the properties of turbulent flows in compressible helical turbulence. The physical mechanism may provide some theoretical suggestions for modeling the compressible helical turbulence.

\section{ACKNOWLEDGMENTS}

This work was supported by the National Key Research and Development Program of China (2019YFA0405300, 2016YFA0401200), the National Natural Science Foundation of China (NSFC Grant No. 91852203), and Science Challenge Project (TZ2016001). The authors thank the National Supercomputer Center in Tianjin (NSCC-TJ) and National Supercomputer Center in Guangzhou (NSCC-GZ) for providing computer time.

\section{APPENDIX: INDETERMINATE PARAMETERS OF EXTERNAL FORCING}

In compressible flows, an alternative expression of external forcing can be constructed by a linear combination of velocity and vorticity as

$$
F_{i}=\sqrt{\rho} \times\left(\pi_{1} u_{i}^{c}+\pi_{2} u_{i}^{s}+\pi_{3} \omega_{i}\right)
$$

where the superscripts $c$ and $s$ denote the compressible and solenoidal components of a physical variable, respectively. The $\pi_{1}, \pi_{2}$, and $\pi_{3}$ are three indeterminate dimensional parameters by the inputting rate of the compressible kinetic energy $\varepsilon_{c}$, the inputting rate of the solenoidal kinetic energy $\varepsilon_{s}$, and the inputting rate of helicity $\varepsilon_{h}$. The external forcing is fixed within the lowest two wave number shells. The inputting rates can be computed through the external forcing as

$$
\begin{aligned}
\varepsilon_{s} & =F_{i}^{s} \cdot v_{i}^{s}=\left(\pi_{1} \sqrt{\rho} u_{i}^{c}+\pi_{2} \sqrt{\rho} u_{i}^{s}+\pi_{3} \sqrt{\rho} \omega_{i}\right)^{s} \cdot(\sqrt{\rho} u)_{i}^{s} \\
& =\pi_{1}\left(\sqrt{\rho} u_{i}^{c}\right)^{s} \cdot(\sqrt{\rho} u)_{i}^{s}+\pi_{2}\left(\sqrt{\rho} u_{i}^{s}\right)^{s} \cdot(\sqrt{\rho} u)_{i}^{s}+\pi_{3}\left(\sqrt{\rho} \omega_{i}\right)^{s} \cdot(\sqrt{\rho} u)_{i}^{s},
\end{aligned}
$$




$$
\begin{gathered}
\varepsilon_{c}=F_{i}^{c} \cdot v_{i}^{c}=\left(\pi_{1} \sqrt{\rho} u_{i}^{c}+\pi_{2} \sqrt{\rho} u_{i}^{s}+\pi_{3} \sqrt{\rho} \omega_{i}\right)^{c} \cdot(\sqrt{\rho} u)_{i}^{c} \\
=\pi_{1}\left(\sqrt{\rho} u_{i}^{c}\right)^{c} \cdot(\sqrt{\rho} u)_{i}^{c}+\pi_{2}\left(\sqrt{\rho} u_{i}^{s}\right)^{c} \cdot(\sqrt{\rho} u)_{i}^{c}+\pi_{3}\left(\sqrt{\rho} \omega_{i}\right)^{c} \cdot(\sqrt{\rho} u)_{i}^{c}, \\
\varepsilon_{h}=2 * F_{i} / \sqrt{\rho} \cdot \omega_{i}=\left(\pi_{1} \sqrt{\rho} u_{i}^{c}+\pi_{2} \sqrt{\rho} u_{i}^{s}+\pi_{3} \sqrt{\rho} \omega_{i}\right) \cdot \omega_{i}=\pi_{1} u_{i}^{s} \cdot \omega_{i}+\pi_{3} \omega_{i} \cdot \omega_{i} .
\end{gathered}
$$

If we define $a_{11}=\left\langle\left(\sqrt{\rho} u_{i}^{c}\right)^{s} \cdot(\sqrt{\rho} u)_{i}^{s}\right\rangle, a_{12}=\left\langle\left(\sqrt{\rho} u_{i}^{s}\right)^{s} \cdot(\sqrt{\rho} u)_{i}^{s}\right\rangle, \quad a_{13}=\left\langle\left(\sqrt{\rho} \omega_{i}\right)^{s} \cdot(\sqrt{\rho} u)_{i}^{s}\right\rangle$, $a_{21}=\left\langle\left(\sqrt{\rho} u_{i}^{c}\right)^{c} \cdot(\sqrt{\rho} u)_{i}^{c}\right\rangle, a_{22}=\left\langle\left(\sqrt{\rho} u_{i}^{s}\right)^{c} \cdot(\sqrt{\rho} u)_{i}^{c}\right\rangle, a_{23}=\left\langle\left(\sqrt{\rho} \omega_{i}\right)^{c} \cdot(\sqrt{\rho} u)_{i}^{c}\right\rangle, a_{31}=\left\langle u_{i}^{s} \cdot \omega_{i}\right\rangle$, $a_{32}=0, a_{33}=\left\langle\omega_{i} \cdot \omega_{i}\right\rangle$, where $\langle\cdot\rangle$ denotes ensemble average within the lowest two shells, and the above equations can be rewritten as the following form:

$$
\left[\begin{array}{lll}
a_{11} & a_{12} & a_{13} \\
a_{21} & a_{22} & a_{23} \\
a_{31} & a_{32} & a_{33}
\end{array}\right]\left[\begin{array}{l}
\pi_{1} \\
\pi_{2} \\
\pi_{3}
\end{array}\right]=\left[\begin{array}{l}
\varepsilon_{s} \\
\varepsilon_{c} \\
\varepsilon_{h}
\end{array}\right]
$$

Hence, the three indeterminate dimensional parameters can be obtained by solving the above system of linear equations, and their final expression are

$$
\begin{gathered}
\pi_{1}=\left(a_{22} a_{33} \varepsilon_{s}-a_{12} a_{33} \varepsilon_{c}+\left(a_{12} a_{23}-a_{13} a_{22}\right) \varepsilon_{h}\right) * \text { coeff } \\
\pi_{2}=-\left[\left(a_{21} a_{33}-a_{23} a_{31}\right) \varepsilon_{s}-\left(a_{11} a_{33}-a_{13} a_{31}\right) \varepsilon_{c}+\left(a_{11} a_{23}-a_{13} a_{21}\right) \varepsilon_{h}\right] * \text { coeff } \\
\pi_{3}=-\left[a_{22} a_{31} \varepsilon_{s}-a_{12} a_{22} \varepsilon_{c}-\left(a_{11} a_{22}-a_{12} a_{21}\right) \varepsilon_{h}\right] * \text { coeff }
\end{gathered}
$$

where

$$
\text { coeff }=\frac{1}{a_{11} a_{22} a_{33}-a_{12} a_{21} a_{33}+a_{12} a_{23} a_{31}-a_{13} a_{22} a_{31}} .
$$

[1] J. W. Armstrong, B. J. Rickett, and S. R. Spangler, Electron density power spectrum in the local interstellar medium, Astrophys. J. 443, 209 (1995).

[2] H. Xu, H. Li, D. C. Collins, S. Li, and M. L. Norman, Turbulence and dynamo in galaxy cluster medium: Implications on the origin of cluster magnetic fields, Astrophys. J. 698, L14 (2009).

[3] T. Sanada, Helicity Production in the Transition to Chaotic Flows Simulated by Navier-Stokes Equation, Phys. Rev. Lett. 70, 3035 (1993).

[4] B. Qu, A. Naso, and W. J. T. Bos, Cascades of energy and helicity in axisymmetric turbulence, Phys. Rev. Fluid 3, 014607 (2018).

[5] R. Betchov, Semi-isotropic turbulence and helicoidal flows, Phys. Fluids 4, 925 (1961).

[6] H. K. Moffatt and A. Tsinober, Helicity in laminar and turbulence flow, Annu. Rev. Fluid Mech. 24, 281 (1992).

[7] H. K. Moffatt, Degree of knottedness of tangled vortex lines, J. Fluid Mech. 35, 117 (1969).

[8] M. W. Scheeler, W. M. van Rees, H. Kedia, D. Kleckner, and W. T. M. Irvine, Complete measurement of helicity and its dynamics in vortex tubes, Science 357, 487 (2017).

[9] J. J. Moreau, Constantes d'un îlôt tourbillonnaire en fluide parfait barotrope, C. R. hebd. séances Acad. Sci. Paris 252, 2810 (1961).

[10] H. K. Moffatt, Helicity, C. R. Mech. 346, 165 (2018).

[11] A. Pouquet and P. D. Mininni, The interplay between helicity and rotation in turbulence: Implications for scaling laws and small-scale dynamics, Philos. Trans. R. Soc. London, Ser. A 368, 1635 (2010).

[12] D. Serre, Invariants et dégénérescence symplectique de l'équation d'euler des fluides parfaits incompressibles, C. R. hebd. séances Acad. Sci. Paris, Ser. A 298, 349 (1984).

[13] D. Serre, Helicity and other conservation laws in perfect fluid motion, C. R. Mec. 346, 175 (2018).

[14] J. C. Andre and M. Lesieur, Influence of helicity on the evolution of isotropic turbulence at high Reynolds number, J. Fluid Mech. 81, 187 (1977). 
[15] A. Brissaud, U. Frisch, J. Leorat, A. Lesieur, and M. Mazure, Helicity cascades in fully developed isotropic turbulence, Phys. Fluids 16, 1366 (1973).

[16] Q. Chen, S. Chen, and G. L. Eyink, The joint cascade of energy and helicity in three-dimensional turbulence, Phys. Fluids 15, 361 (2003).

[17] A. Alexakis and L. Biferale, Cascades and transitions in turbulent flows, Phys. Rep. 767-769, 1 (2018).

[18] P. D. Ditlevsen and P. Giuliani, Dissipation in helical turbulence, Phys. Rev. E 63, 036304 (2001).

[19] S. Kurien, M. A. Taylor, and T. Matsumoto, Cascade time scales for energy and helicity in homogeneous isotropic turbulence, Phys. Rev. E 69, 066313 (2004).

[20] Y. Li, C. Meneveau, S. Chen, and G. L. Eyink, Subgrid-scale modeling of helicity and energy dissipation in helical turbulence, Phys. Rev. E 74, 026310 (2006).

[21] C. Yu, R. Hong, Z. Xiao, and S. Chen, Subgrid-scale eddy viscosity model for helical turbulence, Phys. Fluids 25, 095101 (2013).

[22] H. Zhou, X. Li, H. Qi, and C. Yu, Subgrid-scale model for large-eddy simulation of transition and turbulence in compressible flows, Phys. Fluids 31, 125118 (2019).

[23] N. Yokoi and A. Yoshizawa, in Progress in Turbulence VII, edited by R. Örlü, T. Alessandro, O. Martin, and P. Joachim, Springer Proceedings in Physics Vol. 196 (Springer, New York, 2017), pp. 115-121.

[24] V. I. Arnold and B. A. Khesin, Topological Methods in Hydrodynamics (Springer Science \& Business Media, New York, 1999).

[25] B. A. Khesin and Y. V. Chekanov, Invariants of the Euler equations for ideal or barotropic hydrodynamics and superconductivity in $D$ dimensions, Physica D 40, 119 (1989).

[26] H. Aluie, Compressible Turbulence: The Cascade and its Locality, Phys. Rev. Lett. 106, 174502 (2011).

[27] H. Aluie, S. Li, and H. Li, Conservative cascade of kinetic energy in compressible turbulence, Astrophys. J. Lett. 751, L29 (2012).

[28] H. Aluie, Scale decomposition in compressible turbulence, Physica D 247, 54 (2013).

[29] G. L. Eyink and T. D. Drivas, Cascades and Dissipative Anomalies in Compressible Fluid Turbulence, Phys. Rev. X 8, 011022 (2018).

[30] Z. Yan, X. Li, J. Wang, and C. Yu, Effect of pressure on joint cascade of kinetic energy and helicity in compressible helical turbulence, Phys. Rev. E 99, 033114 (2019).

[31] F. Waleffe, The nature of triad interactions in homogeneous turbulence, Phys. Fluids A 4, 350 (1992).

[32] L. Biferale, S. Musacchio, and F. Toschi, Inverse Energy Cascade in Three-Dimensional Isotropic Turbulence, Phys. Rev. Lett. 108, 164501 (2012).

[33] L. Biferale, S. Musacchio, and F. Toschi, Split energy-helicity cascades in three-dimensional homogeneous and isotropic turbulence, J. Fluid Mech. 730, 309 (2013).

[34] A. Alexakis, Helically decomposed turbulence, J. Fluid Mech. 812, 752 (2017).

[35] G. Sahoo, F. Bonaccorso, and L. Biferale, Role of helicity for large- and small-scale turbulent fluctuations, Phys. Rev. E 92, 051002(R) (2015).

[36] E. Deusebio and E. Lindborg, Helicity in the Ekman boundary layer, J. Fluid Mech. 755, 654 (2014).

[37] M. Kurgansky, Helicity in dynamic atmospheric processes, Izv. Atmos. Ocean. Phys. 53, 127 (2017).

[38] Z. Yan, X. Li, Y. Fu, and C. Yu, The effect of helicity on kinetic energy cascade in compressible helical turbulence, Adv. Appl. Math. Mech. 11, 700 (2019).

[39] D. Block, I. Teliban, F. Greiner, and A. Piel, Prospects and limitations of conditional averaging, Phys. Scr. 2006, 25 (2006).

[40] L. S. G. Kovasznay, V. Kibens, and R. F. Blackwelder, Large-scale motion in the intermittent region of a turbulent boundary layer, J. Fluid Mech. 41, 283 (1970).

[41] A. S. Teimurazov, R. A. Stepanov, M. K. Verma, S. Barman, A. Kumar, and S. Sadhukhan, Direct numerical simulation of homogeneous isotropic helical turbulence with the TARANG code, J. Appl. Mech. Tech. Phys. 59, 1279 (2018).

[42] W. Sutherland, The viscosity of gases and molecular force, Philos. Mag. Ser. 5 36, 507 (1893).

[43] J. Wang, L.-P. Wang, Z. Xiao, Y. Shi, and S. Chen, A hybrid numerical simulation of isotropic compressible turbulence, J. Comput. Phys. 229, 5257 (2010).

[44] S. K. Lele, Compact finite difference schemes with spectral-like resolution, J. Comput. Phys. 103, 16 (1992). 
[45] D. S. Balsara and C. W. Shu, Monotonicity preserving weighted essentially non-oscillatory schemes with increasingly high order of accuracy, J. Comput. Phys. 160, 405 (2000).

[46] T. Passot, E. Vazquez-Semadeni, and A. Pouquet, A turbulent model for the interstellar medium. II. Magnetic fields and rotation, Astrophys. J. 455, 536 (1995).

[47] R. Samtaney, D. I. Pullin, and B. Kosović, Direct numerical simulation of decaying compressible turbulence and shocklet statistics, Phys. Fluids 13, 1415 (2001).

[48] J. Bec and K. Khanin, Burgers turbulence, Phys. Rep. 447, 1 (2007).

[49] D. A. Donzis and K. R. Sreenivasan, The bottleneck effect and the Kolmogorov constant in isotropic turbulence, J. Fluid Mech. 657, 171 (2010).

[50] V. Borue and S. A. Orszag, Spectra in helical three-dimensional homogeneous isotropic turbulence, Phys. Rev. E 55, 7005 (1997).

[51] J. Wang, T. Gotoh, and T. Watanabe, Spectra and statistics in compressible isotropic turbulence, Phys. Rev. Fluid 2, 013403 (2017).

[52] Z. Yan, X. Li, C. Yu, J. Wang, and S. Chen, Dual channels of helicity cascade in turbulent flows, J. Fluid Mech. 894, R2 (2020).

[53] A. M. Oboukhov, Structure of the temperature field in turbulent flows, Izv. Akad. Nauk SSSR Geogr. Geofiz. 13, 58 (1949).

[54] S. Corrsin, On the spectrum of isotropic temperature fluctuations in an isotropic turbulence, J. Appl. Phys. 22, 469 (1951).

[55] K. R. Sreenivasan, The passive scalar spectrum and the Obukhov-Corrsin constant, Phys. Fluids 8, 189 (1996).

[56] M. P. Martin, U. Piomelli, and G. V. Candler, Subgrid-scale models for compressible large-eddy simulations, Theoret. Comput. Fluid Dyn. 13, 361 (2000).

[57] J. Wang, Y. Shi, L. P. Wang, Z. Xiao, X. T. He, and S. Chen, Effect of compressibility on the small-scale structures in isotropic turbulence, J. Fluid Mech. 713, 588 (2012).

[58] J. Wang, M. Wan, S. Chen, and S. Chen, Kinetic energy transfer in compressible isotropic turbulence, J. Fluid Mech. 841, 581 (2018).

[59] G. L. Eyink, Locality of turbulent cascades, Physica D 207, 91 (2005).

[60] H. Miura and S. Kida, Acoustic energy exchange in compressible turbulence, Phys. Fluids 7, 1732 (1995).

[61] M. V. Melander and F. Hussain, Polarized vorticity dynamics on a vortex column, Phys. Fluids A 5, 1992 (1993).

[62] M. Lesieur, Turbulence in Fluids (Kluwer Academic Publishers, Dordrecht, 1997).

[63] D. Kuzzay, O. Alexandrova, and L. Matteini, Local approach to the study of energy transfers in incompressible magnetohydrodynamic turbulence, Phys. Rev. E 99, 053202 (2019). 